\title{
COMPONENT OBJECT MODELING FOR BEAM PHYSICS PROBLEMS
}

\author{
S.N.Andrianov*, SPbSU, S.Petersburg, Russia
}

\begin{abstract}
In recent years sufficient success was achieved in the modeling and optimization of beamlines on the base of highorder maps. In this paper a new approach based on symbolic representation of high-order aberrations in matrix forms is described. We discuss all the pros and cons of such approach.
\end{abstract}

\section{INTRODUCTION}

In recent years many accelerator design and simulation codes was developed. The most of them are based on numerical simulation all steps of modeling process. Among them we should mention the works by J.Jrwin, Y.T.Yan and their colleagues (for example, [1]), the work by E.Forest, K.Hirata [2] and the work of Leo Michelotti [3], M.Berz [4]. Besides these there are several works devoted to the programming design using modern achievements in software (see, for example, [5]-[6]). Unfortunately, the problems of formalizing of the tasks of beam physics in many respects remain unsolved. Their solution would ensure a capability to store knowledge obtained as results of computing experiments, to fill up the knowledge base with new knowledge without destruction of its structure, to use the similar objects for the solution of the different problems arising in beam physics. On the one hand, the development of the software allows to introduce into modeling practice symbolic calculations (methods and codes of computer algebra), modern ideas of object-problem modeling. On the other hand we witness an enormous growth of capabilities of modern computer technology, for example, parallel computers, which can calculate both in symbolic, and in numerical modes. There are rather many authors who use computer algebra in their investigations. But usually they use computer algebra codes as a powerful calculator. It is necessary to mention the works of B.Autin and his colleagues who try to use computer algebra methods and codes maximal widely [8]. In this paper we suggest a new approach which is based on symbolic representation of necessary information on the one hand and object-oriented design approach on the other hand. These two sides of modeling process allows to create flexible and powerful codes for particle beam study including high order aberrations and space-charge forces. For this purpose we use Component Object Modeling Technique (COM-technique) which permits to ensure for wide classes of beam physics problems

\footnotetext{
*Serge.Andrianov@pobox.spbu.ru
}

necessary flexibility and efficiency of calculations, and simultaneously to store knowledge during usage of the offered COM-technique.

\section{BASIC CONCEPTS AND IDEAS OF THE COM - TECHNIQUE}

The object-oriented design based on the usage of objectoriented models for design the program systems and their components. Speaking about object-oriented design, we mean:

- Object-oriented methodologies (technology) of designing program systems.

- The tools maintaining these technologies.

The object-oriented design can begin at the very first phase of biotic cycle; it is irrelevant what the programming language will be used for implementation of the developed program system: this language can and to not be object-oriented. During the development cycle the objects are some formal designs (for example, quadrupole as physical object) bound with their future implementation on one of the programming languages. The objectoriented software engineering is related to application of object-oriented methodologies (technologies). Usually these object-oriented methodologies are supported by tool software, but even without it they are useful, because they allow to understand different aspects and properties of the developed program system which essentially facilitates implementation, testing, tracking, and designing of the new versions and more essential modification.

\subsection{Database of Physical Control Elements}

So, before the development of the software we should create the structured system of elementary objects, which will allow us to create computer model for the fulfillment of this or that task. Outgoing from the designer's purposes of beamline systems, it is necessary to create database of physical control elements. The point is that for the different problems even the concept such a simple object as a drift will essentially differs. As a basic element for any control element we shall consider so-called ideal object: a ideal drift, ideal quadrupole and so on. The subsequent complication of control elements is implemented on the unified information frame by addition of new properties. Here we deal with inheritance paradigm which is already known 
from the theory of object-oriented design. If necessary each object of this constructed database is accompanied by the help information ensuring its interactive usage. At this phase the designer deals with the only physical information permitting him purposive to manipulation of control elements from the database and to create the personal project. If it is necessary to have on a designer's desktop the structural map of a constructed system. Such map can be built on a computer display or in an iconic kind, or in a selected scale which geometrical parameters of the objects.

\subsection{Formalization of the Structure Objects Database}

At the second phase of designing the designer addresses to the database of the second level, which contains the information on parameters of units ensuring their operation. Here, first of all, it is necessary to attribute the information on fields generated by these objects. In this approach we use the well known philosophy of a perturbation theory which allows to structure the field information. The fringe fields can be included using virtual (as fringe fields do not exist without element by itself) object. For this purpose we use a database of model functions for fringe fields. The selection such as distributions is determined by a principle of the adequate description of experimental data and efficiency of the mathematical description. As base the piecewise constant approximating of distribution of fields is considered. The selection of a necessary object can be carried out from the database of physical objects. It is necessary to consider a problem of space-charge forces specifically. In this case we use the database of model distributions [5].

\subsection{Motion Equations and Matrix Formalism}

The knowledge of field decomposition in used objects allows us to define the approximation order for the motion equation which can be written in the following form [6]:

$$
\frac{d X}{d s}=\sum_{k=0}^{\infty} \mathbf{P}^{1 k}(s) X^{[k]},
$$

where $\mathbf{P}^{1 k}(s)$ are matrices depending on field and its derivatives values calculated on an electrical axis of a control object. Using of the information of the database of the second level (field of objects) we constraint the database of the third level - database of matrices, which appropriate to used objects. According to the matrix formalism concept the basic objects are the matrices $\mathbf{P}^{11}(s)$, that are transfer matrices for ideal objects with independence from the fields. The matrices $\mathbf{P}^{1 k}(k \geq 2)$ can be calculated for rather wide class of functional dependence from . Thus, objects of the third level database are the matrices $\mathbf{P}^{1 k}$, calculated in symbolic forms using computer algebra codes (for example, $M A P L E V$ ) up to some approximation or$\operatorname{der} N$. Similarly we can construct the other matrices which are necessary for our modeling process [7].

\subsection{The Solutions of Motion Equation}

Following the matrix formalism concept solution of motion equation (1) is searched as

$$
\begin{gathered}
X(s)=\sum_{k=0}^{\infty} \mathbf{M}^{1 k}\left(s \mid s_{0}\right) X^{[k]}, \\
X_{0}=X\left(s_{0}\right), \quad \mathbf{M}^{1 k}=\mathbf{M}^{11} \cdot \mathbf{Q}^{1 k}, k \geq 1 .
\end{gathered}
$$

According to our approach we build the fourth level database. It is necessary to note, that this database, as well as previous, consists of two parts: first contains block matrices $\mathbf{M}^{11}$ — linear transfer matrices for control elements, the second contains block matrices $\mathbf{Q}^{1 k}$, describing aberrations generated in the corresponding control element. Similarly we compute block matrices $\mathbf{M}^{11}, \mathbf{Q}^{1 k}$, describing the contribution of space-charge forces. These matrices depend on model distribution functions of the space-charge in a phase (or configuration) space.

\subsection{Selection of Local Coordinate Systems}

For each control element there is some coordinate system, where the motion equation has a simplest form. Such coordinate system is selected as base one, and in this coordinate system we evaluate both matrix $\mathbf{P}^{1 k}$, and matrix $\mathbf{M}^{11}, \mathbf{Q}^{1 k}$. The transition from local coordinate systems to a global system (used for description of the total beamline system) is realized using transformation matrices of coordinate systems T. Such set of matrices makes content of one of auxiliary databases. In particular, the transfer matrix for a solenoid has an extremely simple form in a rotated coordinate system, therefore such matrix $\mathbf{P}^{1 k}$ and corresponding matrices $\mathbf{M}^{1 k}$ enter in the appropriate database. For implementation of direct calculations we use a homothetic transformation for return to the global coordinate system.

\section{SYMBOLIC OPERATIONS}

For realization of a simulation procedure we should construct as from LEGO blocks a beamline system either in the language of motion equation (using matrix $\mathbf{P}^{1 k}$ ), or in the language of evolution matrices $\mathbf{M}^{1 k}$, describing time evolution of particle beam. If the necessary matrix object is missing in the appropriate database, we should use one of computing modules to calculate a required matrix in the symbolic form and then to update the database. Let's remark, that for this purpose we use the program package (in codes MAPLE V and REDUCE) ensuring the necessary operations with non-commutative objects (for example, for the CBH-formula) and with matrices (using of the extended matrix operations such as Kronecker sums and products). The existing symmetries allow to use a block structure of matrices and for the reduction of matrix dimensions $\mathbf{M}^{1 k}$, that essentially reduces computing costs (especially for the high orders of approximation $N$ ). Let's remark also, that for the research of a long-time evolution of beams there is a necessity to construct evolution matrices 
on large intervals. In this case representation by matrices allows us to decrease temporary costs of calculations essentially. This has allowed to study explicitly enough the processes in extract resonance system [8] and problem of influence of space-charge forces (for example, the problem of a halo formation [9-10]).

\section{COMPUTER EXPERIMENT}

In this paper for realization of the approach described above we use the system of $R A D-D E L P H I$, permitting, on the one hand, to build simply a user interface, and on the other hand, to use the dynamic modeling paradigm [11-12] for computing experiments using prepared databases. Moreover, latest versions of DELPHI allow to implement the client-server technology and thus distributed calculations concepts. The second moment, which it is necessary to point, is related to a capability of wide application of paralleling procedures for calculations. The point is that the matrix formalism admits parallel processing realization naturally as the beam simulation problems can be resolved by manipulation with matrix objects. Thus the parallel calculations can, basically, be performed both in numerical, and in symbolic modes.

\section{REFERENCES}

[1] Y.Cai, M.Donald, J.Jrwin, Y.T.Yan LEGO: A Modular Accelerator Design Code, Proc. of the 1997 Part. Acc. Conf. - PAC'97, Vancouver, Canada, (1998), pp.2583-2585.

[2] E.Forest, K.Hirata A Contemporary Guide to Beam Dynamics, Nat. Lab. for High Energy Physics, KEK Report 9212(1992).

[3] L.Michelotti "MXYZPPLK: a Version of Differential Algebra, Fermi Nat. Lab. Report FN-535 (1990).

[4] M.Berz Differential Algebraic Description of Beam Dynamics to Very High Orders, Part. Acc., 24, 1989, pp.???.

[5] S.Shasharina, J.R.Cary, D.L.Bruhwiler Status of MAPA (Modular Accelerator Physics Analysis) and TECH-X Object-Oriented Accelerator Library, Proc. of the 6th European Acc. Conf., Stockholm, Sweden, 1998, pp.1174-1176.

[6] N.Malytski, R.Talman Unified Accelerator Libraries, Proc. of the 1996 Comp. Acc. Phys. Conf. - CAP'96, Sept. 24-27, Williamsburg, Virginia, USA, eds. J.J.Bisognano, A.A.Mondelli, AIP Conf. Proc. 391, NY, 1997, pp.361-372.

[7] S.Andrianov Dynamic Modeling Paradigm and Computer Algebra, Proc. of the 9th Intern. Conf. "Computational Modelling and Computing in Physics", September 16-21, 1996, D5, 11-97-112, pp.60-65, Dubna, 1997.

[8] B.Autin (ed.), C.Carli, T.D’Amico, O.Grobner, M.Martini, E.Wildner BeamOptics: A Program for Analytical Beam Optics, preprint CERN 98-06, 1998, 129 p.

[9] S.Andrianov, High-Order Optics with Space Charge: Analytical Approach, Proc.of the Sixth European Part. Acc. Conf. - EPAC-98, Stockholm, 22-26 June 1998, Inst.of Phys. Publ., Bristol, UK, pp.1091-1093.

[10] S.Andrianov, N.Edamenko Halo Formation and Control, presented on this Conference.

[11] S.Andrianov Dynamic Modeling in Beam Dynamics, Proc. of the Second Int.Workshop on Beam Dynamics \& Optimization - BDO'95, July 4-8, 1995, St.Petersburg, Russia, St.Petersburg, 1996, pp.25-32.
[12] S.Andrianov, A.Dvoeglazov Beam-Lines Design Codes: Dynamical Modeling Approach, Proc. of the Sixth European Part. Acc. Conf. - EPAC-98, Stockholm, 22-26 June 1998, Inst. of Phys. Publ., Bristol, UK, pp.1150-1152. 